\title{
Erratum: Directional Bistability and Nonreciprocal Lasing with Cold Atoms in a Ring Cavity [Phys. Rev. Lett. 121, 163603 (2018)]
}

B. Megyeri, G. Harvie, A. Lampis, and J. Goldwin $\odot$

(C) (Received 19 September 2019; published 8 October 2019)

DOI: 10.1103/PhysRevLett.123.159901

The gain mechanism responsible for lasing was incorrectly identified as being due to Mollow scattering on the $|F=2\rangle \leftrightarrow\left|F^{\prime}=2^{\prime}\right\rangle$ transition (notation as in the original Letter). Subsequent experiments show that gain arises from Raman scattering between the $|F=1\rangle$ and $|F=2\rangle$ hyperfine ground states, driven by the MOT repump light [1]. The rest of the results and conclusions are unchanged.

[1] G. Harvie, A. Butcher, and J. Goldwin, In situ Raman gain between hyperfine ground states in a potassium magneto-optical trap, Phys. Rev. A 100, 033408 (2019). 